\title{
Complexity of Computing the Shapley Value in Games with Externalities
}

\author{
Oskar Skibski \\ Institute of Informatics, University of Warsaw, Poland \\ oskar.skibski@mimuw.edu.pl
}

\begin{abstract}
We study the complexity of computing the Shapley value in games with externalities. We focus on two representations based on marginal contribution nets (embedded MCnets and weighted MC-nets) and five extensions of the Shapley value to games with externalities. Our results show that while weighted MC-nets are more concise than embedded MC-nets, they have slightly worse computational properties when it comes to computing the Shapley value: two out of five extensions can be computed in polynomial time for embedded MC-nets and only one for weighted MC-nets.
\end{abstract}

\section{Introduction}

Coalitional games are a standard model of cooperation in multi-agent systems (Chalkiadakis, Elkind, and Wooldridge 2011). In the classic form the profit of a coalition is assumed to be independent of the coalitions formed by the other players. However, this simplifying assumption does not hold in many settings. For example, if agents in a system have conflicted goals or limited resources, then coalitions naturally affect each other (Dunne 2005). There are also examples of externalities in economics, in oligopolistic markets in particular, where cooperation of some companies affect the profits of the competitors (Yi 2003). That is why, in the last decade coalitional games with externalities have gained attention both in economic (Kóczy 2018; Abe and Funaki 2017) and AI literature (Rahwan et al. 2012; Michalak et al. 2010a).

Externalities present new challenges both conceptually and computationally. On the conceptual side, it is unclear how to extend most solution concepts to games with externalities. In particular, there are several non-equivalent well-established methods of extending the Shapley value to games with externalities proposed by Pham Do and Norde (2007) (EF-value), McQuillin (2009) (MQ-value), $\mathrm{Hu}$ and Yang (2010) (HY-value), Feldman (1996) (SS-value) and Myerson (1977b) (MY-value). On the computational side, externalities highly increase the size of the game itself.

To cope with the extensive space requirement of games with externalities, three different representations were proposed in the literature. The first two, called embedded $M C$ -

Copyright (c) 2020, Association for the Advancement of Artificial Intelligence (www.aaai.org). All rights reserved.

\begin{tabular}{l|c|c} 
& Embedded MC-nets & Weighted MC-nets \\
\hline MQ-value & in P $\left(^{*}\right)$ & in P (Th.6) \\
EF-value & in P (Th.8) & \#P-complete (Th.9) \\
HY-value & \#P-complete (Th.10) & \#P-complete (Th.10) \\
SS-value & \#P-complete (Th.13) & \#P-complete (Th.13) \\
MY-value & \#P-complete (Th.14) & \#P-complete (Th.14)
\end{tabular}

Table 1: Summary of complexity results for computing extended Shapley value in games represented as embedded and weighted MC-nets. (*) Proved by Michalak et al. (2010a).

nets (Michalak et al. 2010a) and weighted MC-nets (Michalak et al. 2010b), are extensions of the well-known logicbased representation: marginal contribution nets (Ieong and Shoham 2005). For the former representation, Michalak et al. (2010a) proved that one extension of the Shapley value can be computed in polynomial time. For the latter one, only partial results (polynomial results under restrictive additional assumptions) have been proposed for three extensions. More recently, Skibski et al. (2020) proposed a new representation, partition decision trees, and proved that all five extensions of the Shapley value listed above can be computed in polynomial time under this representation.

In this paper, we fill a gap in the literature by determining what is the complexity of computing all the five extensions of the Shapley value in games represented as embedded and weighted MC-nets. Specifically, we show that only two out of five extensions can be computed in polynomial time for embedded MC-nets and only one can be computed in polynomial time for weighted $\mathrm{MC}$-nets (unless $\mathrm{P}=\mathrm{NP}$ ). For all other values we show that computation is \#P-complete (see Table 1).

Interestingly, our results are strongly based on graph theory techniques. Specifically, we show that every embedded/weighted MC-nets rule can be represented as (one or more) graphs and that each extended Shapley value can be expressed as the weighted sum over all proper vertex coloring in these graphs. Building upon these general results, for each value we analyze the resulting weighted sum. That is, we show the MQ-value is a weighted sum over 2-colorings and EF-value-over independent sets in a part of the graph. In turn, SS-value under some assumptions is proved to be equal to the number of matchings in a bipartite graph. 


\section{Preliminaries}

In this section, we introduce basic notation and definitions.

\section{Coalitional games with externalities}

Let $N=\{1, \ldots, n\}$ be a set of $n$ players, which will be fixed throughout the paper. A coalition is any nonempty subset of $N$. The set of all possible partition of $N$ is denoted by $\mathcal{P}$ and the set of all embedded coalitions, i.e., coalitions in partitions, by $E C: E C=\{(S, P): P \in \mathcal{P}, S \in P\}$.

In this paper, by game we mean a coalitional game with externalities in a partition function form: formally, for a fixed set of players, a game is a function that assigns a real value to every embedded coalition: $g: E C \rightarrow \mathbb{R}$. We say that a game has no externalities if the value of every coalition does not depend on the partition, i.e., $g(S, P)=g\left(S, P^{\prime}\right)$ for every coalition $S \subseteq N$ and $(S, P),\left(S, P^{\prime}\right) \in E C$.

A value of a player in a game is a real number that represents player's importance or expected outcome. The Shapley value (Shapley 1953) is defined for games without externalities. Assume $g$ is such a game, i.e., there exists $\hat{g}: 2^{N} \rightarrow \mathbb{R}$ such that $g(S, P)=\hat{g}(S)$ for every $(S, P) \in E C$ and $\hat{g}(\emptyset)=0$. Now, the Shapley value is defined as follows:

$$
S V_{i}(\hat{g})=\sum_{S \subseteq N} \zeta_{S}^{i} \cdot \hat{g}(S),
$$

where $\zeta_{S}^{i}=(|S|-1) !(n-|S|) ! / n$ ! if $i \in S$, and $\zeta_{S}^{i}=$ - $|S| !(n-|S|-1) ! / n !$, otherwise.

Shapley (1953) famously proved that the Shapley value is a unique value that satisfies four simple axioms: Efficiency, Symmetry, Additivity and Null-Player. In games with externalities, however, these classic axioms are too weak to guarantee uniqueness. Hence, numerous extensions of the Shapley value to games with externalities have been proposed (see (Kóczy 2018) for a recent overview), most of the form:

$$
E S V_{i}^{\omega}(g)=\sum_{(S, P) \in E C} \omega_{i}(S, P) \cdot g(S, P)
$$

for some weights $\omega: E C \times N \rightarrow \mathbb{R}$.

We will focus on five such extensions. Four of them can be obtained as the composition of two functions: the first one maps a game with externalities to a game without externalities and the second one applies the original Shapley value. In the simplest definitions, the mapping is obtained by simply taking a value of each coalition from one specific partition:

McQuillin value (MQ-value) (McQuillin 2009) is defined as $M Q_{i}(g)=E S V_{i}^{\omega}(g)$ for $\omega_{i}(S, P)=\zeta_{S}^{i} \cdot[|P| \leq 2]$.

Externality-free value (EF-value) (Pham Do and Norde 2007) is defined as $E F_{i}(g)=E S V_{i}^{\omega}(g)$ for $\omega_{i}(S, P)=$ $\zeta_{S}^{i} \cdot[|P|-1=n-|S|]$.

Here, we used Iverson brackets: $[\varphi]=1$ if statement $\varphi$ is true, and $[\varphi]=0$, otherwise. EF-value was independently proposed by De Clippel and Serrano (2008).

MQ-value and EF-value ignore values of a coalition in most partitions. In turn, HY-value and SS-value for every coalition take a weighted average over all partitions it is in; the difference is HY-value assigns greater weights to partitions with more coalitions, while SS-value-to partitions with larger coalitions:

\begin{tabular}{l|ccccc|c} 
& {$[4]$} & {$[3,1]$} & {$[2,2]$} & {$[2,1,1]$} & {$[1,1,1,1]$} & $\times$ \\
\hline MQ & 1 & 0 & 0 & 0 & 0 & $1 / 30$ \\
EF & 0 & 0 & 0 & 0 & 1 & $1 / 30$ \\
HY & 5 & 10 & 10 & 17 & 26 & $1 / 6090$ \\
SS & 6 & 2 & 1 & 1 & 1 & $1 / 720$ \\
MY & 10 & -6 & -5 & 9 & -24 & $1 / 30$
\end{tabular}

Table 2: Weights $\omega_{i}(S, P)$ for different extended Shapley values with $|N|=6,|S|=2$ and $i \in S$. Columns are labeled with the integer partitions corresponding to sizes of coalitions in $P \backslash\{S\}$. For each value, the last column contains a common multiplier (e.g., for HY-value and $P \backslash\{S\}$ of the form $\{\{j, k\},\{l\},\{m\}\}$ it holds $\left.w_{i}(S, P)=17 / 6090\right)$. Cells most important in our complexity results are marked.

Hu-Yang value (HY-value) (Hu and Yang 2010) is defined as $H Y_{i}(g)=E S V_{i}^{\omega}(g)$ for $\omega_{i}(S, P)=\zeta_{S}^{i} \cdot \theta(S, P) /|\mathcal{P}|$, where $\theta(S, P)=\left|\left\{P^{\prime} \in \mathcal{P}:\left\{T \backslash S: T \in P^{\prime}\right\}=P \backslash\{S\}\right\}\right|$.

Stochastic Shapley value (SS-value) (Feldman 1996) is defined as $S S_{i}(g)=E S V_{i}^{\omega}(g)$ for $\omega_{i}(S, P)=\zeta_{S}^{i}$. $\left(\prod_{T \in P \backslash\{S\}}(|T|-1) !\right) /(n-|S|)$ !.

SS-value was also studied by Macho-Stadler, PérezCastrillo, and Wettstein (2007) and Skibski, Michalak, and Wooldridge (2018).

Finally, Myerson (1977b) studied how the Null-player axiom can be strengthen in order to obtain unique characterization. His analysis led to a value which cannot be interpreted as a composition of two functions mentioned above.

Myerson value (MY-value) (Myerson 1977b)

is defined as $M Y_{i}(g)=E S V_{i}^{\omega}(g)$ for $\omega_{i}(S, P)=$ $(-1)^{|P|}\left(\left(\sum_{T \in P \backslash\{S\}, i \notin T} \frac{(|P|-2) !}{(n-|T|)}\right)-\frac{(|P|-1) !}{n}\right)$

See Table 2 for an illustration of $\omega$ weights.

\section{Representations}

Game represented as a single rule $\gamma$ is denoted by $g^{\gamma}$ and as the set of rules $\Gamma=\left\{\gamma_{1}, \ldots, \gamma_{k}\right\}$ by $g^{\Gamma}$.

\section{MC-nets (Ieong and Shoham 2005)}

Marginal contribution nets (MC-nets) are a representation for games without externalities. The game is represented as a set of MC-nets rules of the form: $(\alpha \rightarrow c)$. Here, $c \in \mathbb{R}$ is the weight of a rule and $\alpha$ is a boolean expression over $N$ of the form:

$$
\left(a_{1}^{+} \wedge \cdots \wedge a_{m}^{+} \wedge \neg a_{1}^{-} \wedge \cdots \wedge \neg a_{l}^{-}\right),
$$

where $a_{1}^{+}, \ldots, a_{m}^{+} \in N$ are called positive literals and $a_{1}^{-}, \ldots, a_{l}^{-} \in N$ are called negative literals. We denote sets of positive and negative literals by $\oplus(\alpha)$ and $\ominus(\alpha)$, respectively, and assume $\oplus(\alpha) \cap \ominus(\alpha)=\emptyset$ and $\oplus(\alpha) \neq \emptyset .^{1} \mathrm{~A}$ coalition $S$ satisfies $\alpha$ if it contains all positive literals and does not contain any negative literal, i.e., $\oplus(\alpha) \subseteq S$ and $\ominus(\alpha) \cap S=\emptyset$. Now, in a game represented as a set of MCnets rules the value of coalition $S$ is the sum of weights of all satisfied rules.

\footnotetext{
${ }^{1}$ Ieong and Shoham (2005) allows rules without positive literals which entails that the empty coalition may have non-zero value. As standard in the literature, we do not allow such situations.
} 
Embedded MC-nets (Michalak et al. 2010a)

An embedded MC-nets rule is of the form:

$$
\left(\alpha_{1} \mid \alpha_{2}, \alpha_{3}, \ldots, \alpha_{k}\right) \rightarrow c,
$$

where $c \in \mathbb{R}$ is the weight of a rule and $\alpha_{1}, \ldots, \alpha_{k}$ are boolean expressions as in Eq. (2). An embedded coalition $(S, P)$ satisfies the rule if $S$ satisfies $\alpha_{1}$ and for every $\alpha_{i}$ with $i>1$ there exists a coalition $T \in P \backslash\{S\}$ that satisfies it. Now, in a game represented as a set of embedded MCnets rules the value of embedded coalition $(S, P)$ is the sum of weights of all satisfied rules.

Weighted MC-nets (Michalak et al. 2010b)

A weighted MC-nets rule is of the form:

$\left(\alpha_{1}^{1} \rightarrow c_{1}^{1}\right) \ldots\left(\alpha_{k_{1}}^{1} \rightarrow c_{k_{1}}^{1}\right)|\ldots|\left(\alpha_{1}^{m} \rightarrow c_{1}^{m}\right) \ldots\left(\alpha_{k_{m}}^{m} \rightarrow c_{k_{m}}^{m}\right)$, where $\left(\alpha_{j}^{i} \rightarrow c_{j}^{i}\right)$ for every $i \in\{1, \ldots, m\}$ and $j \in$ $\left\{1, \ldots, k_{i}\right\}$ is an MC-nets rule. A partition $P$ satisfies the rule if it can be partitioned into $m$ disjoint subsets $P=$ $P_{1} \cup \ldots \dot{\cup} P_{m}$ such that for every $i \in\{1, \ldots, m\}$ and $j \in$ $\left\{1, \ldots, k_{i}\right\}$ rule $\left(\alpha_{j}^{i} \rightarrow c_{j}^{i}\right)$ is satisfied by some coalition from $P_{i}$. Now, in a game represented as a set of weighted MC-nets rules the value of embedded coalition $(S, P)$ is the sum of weights of all MC-nets rules $(\alpha \rightarrow c)$ that $S$ satisfies in all (weighted MC-nets) rules satisfied by $P$.

\section{Graphs}

A graph is a pair $(V, E)$ where $V$ is the set of nodes and $E$ is the set of undirected edges, i.e., subsets of nodes of size 2.

Two nodes are adjacent if there is an edge connecting them. A clique is a subset of nodes every two of which are adjacent. An independent set is a subset of nodes no two of which are adjacent.

A (proper vertex) $k$-coloring of a graph is a function, $f$ : $V \rightarrow\{1, \ldots, k\}$, that assigns colors $\{1, \ldots, k\}$ to nodes in a way that every two adjacent nodes have different colors, i.e., $f(v) \neq f(u)$ for every $\{v, u\} \in E$. In other words, nodes colored with the same color are an independent set. The set of all $k$-colorings of a graph $G$ is denoted by $C_{k}(G)$.

A $k$-coloring $f$ results in the partition of nodes:

$$
P_{f}=\left\{f^{-1}(i): i \in\{1, \ldots, k\}, f^{-1}(i) \neq \emptyset\right\} .
$$

A set in $P_{f}$ that contains node $v_{i}$ is denoted by $S_{f}^{i}$. We say that $f$ uses exactly $p$ colors if $\left|P_{f}\right|=p$. Finally, we will denote by $\theta(f)$ the number of all $k$-colorings that result in the partition $P_{f}$; note that $\theta(f)=k(k-1) \cdots\left(k-\left|P_{f}\right|+1\right)$.

We will consider graphs labeled with subsets of players: $l: V \rightarrow 2^{N}$. We will denote the label of node $v$ by $l(v)$ and define $l(U)=\bigcup_{v \in U} l(v)$ for a subset of nodes $U \subseteq V$ and $l(P)=\{l(U): U \in P\}$ for a partition $P$ of nodes $V$.

Example 1. Figure 1 presents an example graph $G=$ $(V, E)$ and a 4-coloring $f$. Coloring $f$ results in the following partition of nodes: $P_{f}=\left\{\left\{v_{1}, v_{4}\right\},\left\{v_{2}\right\},\left\{v_{3}\right\}\right\}$ (there are $\theta(f)=4 \cdot 3 \cdot 2$ different 4-colorings that result in this partition). We have $S_{f}^{1}=\left\{v_{1}, v_{4}\right\}$.

Note that labels of nodes in $G$ form a partition of the set of players $N=\{1, \ldots, 6\}:\left\{l\left(v_{1}\right), l\left(v_{2}\right), l\left(v_{3}\right), l\left(v_{4}\right)\right\} \in \mathcal{P}$. Hence, also $l\left(P_{f}\right)$ is a partition of $N$ and $\left(l\left(S_{f}^{1}\right), l\left(P_{f}\right)\right)=$ $(\{1,2,6\},\{\{1,2,6\},\{3,5\},\{4\}\})$ is an embedded coalition.

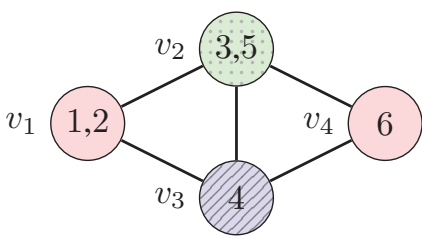

Figure 1: Graph $G=(V, E)$ and a 4-coloring $f$ with colors: 1 (blue/striped), 2 (yellow/checked), 3 (red/plain), 4 (green/dotted). Note that color 2 is not used.

\section{Mapping MC-Nets into Graphs}

The goal of this section is to show that (1) every embedded and weighted MC-nets rule can be represented as (one or more) graphs, and (2) a game represented as embedded and weighted MC-nets can be defined based on (proper vertex) colorings in such graphs.

To this end, we begin by introducing a subclass of weighted MC-nets rules under the name hybrid rules. The name comes from the fact that hybrid rules, while they are formally weighted MC-nets rules, have a form almost identical to embedded MC-nets rules.

Definition 1. (Hybrid rules) A hybrid rule is a weighted MC-nets rule with $m=1$ of the form:

$$
\gamma=\left(\alpha_{1} \rightarrow c\right)\left(\alpha_{2} \rightarrow 0\right) \ldots\left(\alpha_{k} \rightarrow 0\right),
$$

with $\oplus\left(\alpha_{i}\right) \cap \oplus\left(\alpha_{j}\right)=\emptyset$ for $i, j \in\{1, \ldots, k\}, i \neq j$ and $\bigcup_{i=1}^{k} \oplus\left(\alpha_{i}\right)=N$. We will call $c \in \mathbb{R}$ the weight of rule $\gamma$.

Note that for every hybrid rule $\left\{\oplus\left(\alpha_{1}\right), \ldots, \oplus\left(\alpha_{k}\right)\right\}$ is a partition of $N$. Based on the definition of weighted MC-nets, embedded coalition $(S, P)$ satisfies hybrid rule $\gamma$ if $S$ satisfies $\alpha_{1}$ and for every $\alpha_{i}$ with $i>1$ there exists a coalition in $P$ that satisfies it (note that, unlike embedded MC-nets, $S$ may also satisfy $\alpha_{i}$ for $i>1$ ).

In Lemma 1, we show that every weighted MC-nets rule can be expressed using polynomially many hybrid rules.

Lemma 1. Every weighted $M C$-nets rule of size $S$ is equivalent to a set of hybrid rules of size poly $(n, S)$.

In turn, in Lemma 2, we show that embedded MC-nets rules are equivalent to a subset of hybrid rules. We will use a notion of compatibility: we say that expressions $\alpha_{i}, \alpha_{j}$ are compatible, denoted by $\operatorname{comp}\left(\alpha_{i}, \alpha_{j}\right)$, if there exists a coalition that satisfies both of them, i.e., if $\left(\oplus\left(\alpha_{i}\right) \cup \oplus\left(\alpha_{j}\right)\right) \cap$ $\left(\ominus\left(\alpha_{i}\right) \cup \ominus\left(\alpha_{j}\right)\right)=\emptyset$.

Lemma 2. Every embedded MC-nets rule of size $S$ is equivalent to a hybrid rule of size $\operatorname{poly}(n, S)$ satisfying:

$$
\begin{aligned}
& \forall_{1<i, j \leq k}\left(\left(\operatorname{comp}\left(\alpha_{1}, \alpha_{i}\right) \wedge \operatorname{comp}\left(\alpha_{1}, \alpha_{j}\right)\right) \rightarrow\right. \\
& \quad\left(\operatorname{comp}\left(\alpha_{i}, \alpha_{j}\right) \wedge\left(\left|\oplus\left(\alpha_{i}\right)\right|=\left|\oplus\left(\alpha_{j}\right)\right|=1\right)\right) .
\end{aligned}
$$

Moreover, every hybrid rule of size $S$ satisfying $(*)$ is equivalent to an embedded $M C$-nets rule of size $\operatorname{poly}(n, S)$.

Lemmas 1 and 2 will be crucial in our complexity analysis as they allow us to focus on hybrid rules.

So far, we have shown the mapping from weighted MCnets and embedded MC-nets rules to hybrid rules. In what follows, we show that every hybrid rule can be represented as a graph. 
Definition 2. (Graph $G^{\gamma}$ ) For a hybrid rule $\gamma$, graph $G^{\gamma}=(V, E)$ is a graph where nodes represent expressions $\alpha_{1}, \ldots, \alpha_{k}$ and are labeled with sets $\oplus\left(\alpha_{1}\right), \ldots, \oplus\left(\alpha_{k}\right)$ and edges connect incompatible expressions $\alpha_{i}, \alpha_{j}$ :

- $V=\left\{v_{1}, \ldots, v_{k}\right\}$ with $l\left(v_{i}\right)=\oplus\left(\alpha_{i}\right)$ for every $v_{i} \in V$;

- $E=\left\{\left\{v_{i}, v_{j}\right\} \subseteq V: \neg \operatorname{comp}\left(\alpha_{i}, \alpha_{j}\right)\right\}$.

We note that a similar construction of a graph for the righthand side part of the embedded MC-nets was proposed by Skibski et al. (2016).

Example 2. Consider a hybrid rule $\gamma=\left(\alpha_{1} \rightarrow 1\right)\left(\alpha_{2} \rightarrow\right.$ $0)\left(\alpha_{3} \rightarrow 0\right)\left(\alpha_{4} \rightarrow 0\right)$ with:

$$
\begin{array}{ll}
\alpha_{1}=(1 \wedge 2 \wedge \neg 3), & \alpha_{2}=(3 \wedge 5), \\
\alpha_{3}=(4 \wedge \neg 1 \wedge \neg 3 \wedge \neg 6), & \alpha_{4}=(6 \wedge \neg 5) .
\end{array}
$$

Note that $\operatorname{comp}\left(\alpha_{1}, \alpha_{4}\right)$ and $\neg \operatorname{comp}\left(\alpha_{i}, \alpha_{j}\right)$ for other $i, j \in$ $\{1, \ldots, 4\}, i \neq j$. Graph $G^{\gamma}$ is depicted in Figure 1.

Since $\alpha_{4}$ is the only expression compatible with $\alpha_{1}$ (other than $\alpha_{1}$ itself) and $\left|\oplus\left(\alpha_{4}\right)\right|=1$, we get that $\gamma$ satisfies condition $(*)$. Hence, from Lemma 2 we know that it is equivalent to some embedded MC-nets rule. One such a rule is: $(1 \wedge 2) \mid(3 \wedge 5 \wedge \neg 6)(4 \wedge \neg 3 \wedge \neg 6) \rightarrow 1$.

Definition 2 shows that every hybrid rule can be represented as a graph in which labels of nodes form a partition of players. The natural question arises: does every such graph represent some hybrid rule? The answer is "yes", which we prove in the following lemma.

Lemma 3. For a graph $G=(V, E)$ with labels $l$ there exists a hybrid rule $\gamma$ such that $G=G^{\gamma}$ if and only if $\{l(v): v \in$ $V\}$ is a partition of $N$ of size $|V|$.

The next lemma states the necessary and sufficient conditions for the graph to represent a hybrid rule that satisfies condition $(*)$.

Lemma 4. For a graph $G=(V, E)$ with labels $l$ there exists a hybrid rule $\gamma$ satisfying condition $(*)$ such that $G=G^{\gamma}$ if and only if:

(i) $\{l(v): v \in V\}$ is a partition of $N$ of size $|V|$;

(ii) $\left\{u \in V \backslash\left\{v_{1}\right\}:\left\{v_{1}, u\right\} \notin E\right\}$ (nodes not adjacent to $\left.v_{1}\right)$ form an independent set; and

(iii) $|l(u)|=1$ for every node $u$ not adjacent to $v_{1}\left(u \neq v_{1}\right)$.

Based on Lemmas 1-4 we know that every weighted MCnets rule can be represented as (one or more) graphs and every embedded MC-nets rule can be represented as a graph satisfying conditions (i)-(iii).

Let us now explain how a game represented as a hybrid rule $\gamma$ can be defined based on graph $G^{\gamma}$. Fix a hybrid rule $\gamma$ and consider a partition $P$ that satisfies it. Since every node in graph $G^{\gamma}$ is labeled with a set of players which is equal to the set of positive literals in some expression $\alpha_{i}$, it is clear that all these players must appear in the same coalition in $P$. This observation combined with the fact that every player appears in exactly one node implies that $P$ can be associated with a partition of nodes in graph $G^{\gamma}$.

Consider partition $P^{*}$ of nodes that correspond to $P$. Note that two adjacent nodes cannot belong to the same coalition in $P^{*}$, because they represent incompatible expressions that cannot be satisfied by one coalition. Hence, every set in $P^{*}$ is an independent set. In result, we get that $P^{*}$ corresponds to some coloring of a graph.

This analysis is formalized in the following lemma.

Lemma 5. Partition $P$ satisfies a hybrid rule $\gamma$ if and only if there exists $k$-coloring $f \in C_{k}\left(G^{\gamma}\right)$ such that $P=l\left(P_{f}\right)$. Moreover:

$$
g^{\gamma}(S, P)=c \cdot\left[\exists_{f \in C_{k}\left(G^{\gamma}\right)}\left(P=l\left(P_{f}\right) \wedge S=l\left(S_{f}^{1}\right)\right)\right] .
$$

Example 3. Consider a hybrid rule $\gamma$ from Example 2 with graph $G^{\gamma}$ depicted in Figure 1. Let us discuss all possible 4-colorings of $G^{\gamma}$ :

- There are no colorings of $G^{\gamma}$ that use 2 or 3 colors.

- There are 24 colorings that use 3 colors: $f\left(v_{1}\right)=f\left(v_{4}\right)=$ $a, f\left(v_{2}\right)=b$ and $f\left(v_{3}\right)=c$ where $a, b, c \in\{1, \ldots, 4\}$ are different colors. Note that for every such coloring $f$ we have: $P_{f}=\{\{1,2,6\},\{3,5\},\{4\}\}$.

- There are 24 colorings that use 4 colors; in these colorings all nodes have different colors. For every such coloring $f$ we get: $P_{f}=\{\{1,2\},\{3,5\},\{4\},\{6\}\}$

Overall, 48 colorings results in two partitions of players. Now, from Lemma 5, game $g^{\gamma}$ is defined as follows:

$$
g^{\gamma}(\{1,2,6\},\{\{1,2,6\},\{3,5\},\{4\}\})=1,
$$$$
g^{\gamma}(\{1,2\},\{\{1,2\},\{3,5\},\{4\},\{6\}\})=1,
$$

and $g^{\gamma}(S, P)=0$ for the remaining embedded coalitions.

In the following section, we consider computing extended Shapley values in games defined with Eq. (3).

\section{Computing extended Shapley values}

From Eq. (1) we know that all extended Shapley values considered by us satisfy linearity, i.e., $\operatorname{ESV}\left(g+g^{\prime}\right)=$ $E S V(g)+E S V\left(g^{\prime}\right)$ and $E S V(c \cdot g)=c \cdot E S V(g)$ for every two games $g, g^{\prime}$ and $c \in \mathbb{R}$. Thus, in our computational analysis we can focus on games represented as a single rule and, based on Lemmas 1 and 2, as a single hybrid rule. Moreover, we can assume the weight of this rule is 1 (i.e., $c=1$ ). Hence, from now on, we will assume that game is represented as a hybrid rule with weight 1 .

Fix such a hybrid rule $\gamma$. In Lemma 5, we showed that the value of an embedded coalition $(S, P)$ is non-zero if and only if there exists a coloring $f$ in graph $G^{\gamma}$ such that $P=P_{f}$. Since there are $\theta(f)$ colorings that results in the same partition as $f$, from Lemma 5 and Eq. (1) we get the following formula for extended Shapley values:

$$
E S V_{i}\left(g^{\gamma}\right)=\sum_{f \in C_{k}\left(G^{\gamma}\right)} \omega_{i}\left(l\left(S_{f}^{1}\right), l\left(P_{f}\right)\right) / \theta(f) .
$$

To put it in words, extended Shapley value in game $g^{\gamma}$ is a weighted sum over all colorings in graph $G^{\gamma}$. Weights depend on $l\left(P_{f}\right)$ (partition of players resulting from the coloring $f$ ), $l\left(S_{f}^{1}\right)$ (union of labels of all nodes colored with the same color as node $v_{1}$ ), and player $i \in N$.

More generally, we can consider the following counting problem that we name WeIGHTED Coloring Counting. The problem is parametrized with weights $\omega^{*}: C_{k}(G) \rightarrow \mathbb{R}$ that for each coloring assigns some real value. 
Definition 3. $\omega^{*}$-Weighted COLORING COUNTING Input: graph $G=(V, E)$, labels $l: V \rightarrow 2^{N}$ s.t. $\{l(v): v \in$ $V\}$ is a partition of players $N$

Output: $\sum_{f \in C_{|V|}(G)} \omega^{*}(f)$.

This problem in general is computationally challenging, as it generalizes the problem of counting all $k$-colorings which is \#P-complete and allows us to determine whether a graph is 3-colorable which is NP-complete.

Based on Eq. (4), computing each extended Shapley value for a fixed player can be considered a special case of Weighted Coloring Counting. In the following sections, we analyze these problems one by one. Values are ordered in ascending order by the complexity of their formula:

- First two values, MQ-value and EF-value, take into account only one partition $P$ for every coalition $S$; hence, they can be computed by traversing all subsets, not all partitions of players.

- In HY-value, considered third, the weight of an embedded coalition $(S, P)$ depends solely on $|S|$ and $|P|$; this allows us to group all colorings that use the same number of colors.

- Finally, in the last two values, SS-value and MY-value, weights depend on sizes of all coalitions in a partition.

Before we move to the next section, let us roughly explain a technique that we use in the proofs of Theorem 9, 10 and 14. This technique was used in several complexity results for the Shapley value in games without externalities (see, e.g., (Aziz et al. 2009; Michalak et al. 2013)).

Assume we want to compute $x_{1}, \ldots, x_{k}$ and we have an algorithm that computes the sum $f(j)=\sum_{m=1}^{k} a_{j, m} x_{m}$ for some weights $a$ that depend on $m$ and some external parameter $j \in\{1, \ldots, k\}$. To this end, we can construct a system of linear equations with the following matrix form:

$$
\left[\begin{array}{cccc}
a_{1,1} & a_{1,2} & \ldots & a_{1, k} \\
a_{2,1} & a_{2,2} & \ldots & a_{2, k} \\
\vdots & \vdots & \ddots & \vdots \\
a_{k, 1} & a_{k, 2} & \ldots & a_{k, k}
\end{array}\right] \cdot\left[\begin{array}{c}
x_{1} \\
x_{2} \\
\vdots \\
x_{k}
\end{array}\right]=\left[\begin{array}{c}
f(1) \\
f(2) \\
\vdots \\
f(k)
\end{array}\right]
$$

Now, if the matrix $\left(a_{j, m}\right)_{1 \leq j, m \leq k}$ has non-zero determinant, then it is invertible. Hence, if we know $f(1), \ldots, f(k)$, then using Gaussian elimination we can compute $x_{1}, \ldots, x_{k}$.

In our case, $f(j)$ will be an extended Shapley value and $x_{m}$ will be the number of independent sets of size $m$ (Theorem 9), $k$-colorings that use $m$ colors (Theorem 10) or matchings in a bipartite graph of size $m$ (Theorem 14). Hence, based on the fact that computing $\sum_{m=1}^{k} x_{m}$ is \#Phard we will get that computing EF-value, HY-value and MY-value is also \#P-hard.

Note that based on Eq. (4) each extended Shapley value is a sum over exponentially many colorings and, in general, two colorings that result in different partitions of nodes may have different weights. Hence, the main challenge with this approach is to (1) express an extended Shapley value as a weighted sum over polynomial number of elements and (2) creating a system of linear equations that results in a matrix which is invertible.

\section{Computing MQ-value}

We begin with the analysis of MQ-value. Consider Eq. (4) for weights of MQ-value:

$$
M Q_{i}\left(g^{\gamma}\right)=\sum_{f \in C_{k}\left(G^{\gamma}\right)} \frac{\zeta_{l\left(S_{f}^{1}\right)}^{i}}{\theta(f)} \cdot\left[\mid l\left(P_{f}\right) \leq 2\right] .
$$

As we can see, only colorings that use 1 or 2 colors have non-zero weights. It is easy to verify that instead of going through all $k$-colorings it is enough to consider 2 -colorings of a graph. Using the fact that for every 2 -coloring $f$ we have $\theta(f)=2$, we get:

$$
M Q_{i}\left(g^{\gamma}\right)=\frac{1}{2} \sum_{f \in C_{2}\left(G^{\gamma}\right)} \zeta_{l\left(S_{f}^{1}\right)}^{i} .
$$

While in a connected graph there are at most two 2colorings, in a disconnected graph it can be exponentially many. Nevertheless, in Theorem 6, we show that this sum can be computed in polynomial time for every graph.

Theorem 6. For a game represented as weighted MC-nets, $M Q$-value can be computed in polynomial time.

Sketch of proof. From Lemma 1 and linearity of MQ-value we can focus on hybrid rules with weight 1 . Fix such a hybrid rule $\gamma$. We will focus on the simplest case: $i \in l\left(v_{1}\right)$. Let us define a table $T[1 \ldots n]$ as follows: $T[s]=\mid\{f \in$ $\left.C_{2}\left(G^{\gamma}\right):\left|l\left(S_{f}^{1}\right)\right|=s\right\} \mid$. To put it in words, $T[s]$ is the number of 2-colorings of $G^{\gamma}$ in which there are $s$ players in nodes colored with the same color as node $v_{1}$. From Eq. (6) we have:

$$
M Q_{i}\left(g^{\gamma}\right)=\sum_{s=1}^{n}(s-1) !(n-s) ! /(2 n !) \cdot T[s] .
$$

Thus, it is enough to compute table $T$.

Table $T$ can be computed with the following dynamic programming algorithm. Assume $T$ is filled with zeros at the beginning. Let $A_{1}, \ldots, A_{m}$ be connected components of graph $G^{\gamma}$, and let $\left\{B_{j}, C_{j}\right\}$ be a unique partition of $A_{j}$ into two independent sets. Components and partitions can be found by performing several breadth-first searches. If partition $\left\{B_{j}, C_{j}\right\}$ does not exist for at least one component $A_{j}$, then the calculation is complete ( $T$ is a zero table). Assume otherwise and assume $v_{1} \in B_{1}$. We begin by assigning $T\left[\left|l\left(B_{1}\right)\right|\right]=2$, since there are two 2-colorings of $A_{1}$. Now, we consider components $A_{2}, \ldots, A_{m}$, one by one, and for each component consider two cases: either $B_{j}$ or $C_{j}$ is colored with the same color as node $v_{1}$. Thus, in each step, we update table $T$ by replacing it with a new table $T^{\prime}$ defined as follows: $T^{\prime}[s]=T\left[s-\left|l\left(B_{j}\right)\right|\right]+T\left[s-\left|l\left(C_{j}\right)\right|\right]$ for $1 \leq$ $s \leq n$ (assuming $T[s]=0$ for $s \leq 0$ ). After the $m$-th step, the calculation is complete.

Theorem 6 implies polynomial computation also for embedded MC-nets.

Corollary 7. For a game represented as embedded MCnets, $M Q$-value can be computed in polynomial time.

Proof. Directly from Theorem 6. This result was also proved by (Michalak et al. 2010a). 


\section{Computing EF-value}

EF-value, considered by us next, is complementary to MQvalue. Consider Eq. (4) for weights of EF-value:

$$
E F_{i}\left(g^{\gamma}\right)=\sum_{f \in C_{k}\left(G^{\gamma}\right)}\left[\left|P_{f}\right|-1=n-\left|l\left(S_{f}^{1}\right)\right|\right] \cdot \zeta_{l\left(S_{f}^{1}\right)}^{i} / \theta(f) .
$$

Note that condition $\left|P_{f}\right|-1=n-\left|l\left(S_{f}^{1}\right)\right|$ holds if and only if every player $i \in N \backslash l\left(S_{f}^{1}\right)$ form a singleton coalition $\{i\}$ in $l\left(P_{f}\right)$, i.e., node $v$ such that $i \in l(v)$ has a label of size one and is colored with a different color than all other nodes. Hence, $P_{f}$ is uniquely defined by set $S_{f}^{1}$.

Let us analyze the conditions on set $S_{f}^{1}$. Let $V^{*}$ be the set of nodes with non-singleton labels. For an arbitrary set of nodes $S \subseteq V$, there exists a coloring $f$ with non-zero weight such that $S=S_{f}^{1}$ if and only if: (1) $S$ is an independent set; (2) $S$ contains node $v_{1}$; and (3) $S$ contains set $V^{*}$. This implies that if $V^{*} \cup\left\{v_{1}\right\}$ does not form an independent set, then the formula evaluates to zero. Assume otherwise.

We get that $S$ is the union of $V^{*} \cup\left\{v_{1}\right\}$ and an independent set of nodes which are not adjacent to $V^{*} \cup\left\{v_{1}\right\}$. Let us denote these nodes by $U$ (formally, $U=\left\{u \in V \backslash\left(V^{*} \cup\right.\right.$ $\left.\left.\left.\left\{v_{1}\right\}\right):\{u, w\} \in E \rightarrow w \notin V^{*} \cup\left\{v_{1}\right\}\right\}\right)$. Also, if $S=S_{f}^{1}$ for some coloring $f$, then there are $\theta(f)$ colorings with the same set $S_{f}^{1}$. Hence, we get the following formula for EFvalue:

$$
E F_{i}\left(g^{\gamma}\right)=\sum_{S \in I\left(G^{\gamma}\right): S \subseteq U} \zeta_{l\left(S \cup V^{*} \cup\left\{v_{1}\right\}\right)}^{i}
$$

where $I\left(G^{\gamma}\right)$ is the set of all independent sets in graph $G^{\gamma}$.

In the following two theorems, we show that this sum is hard to compute in general, but it is easy to compute if graph satisfies condition stated in Lemma 4.

Theorem 8. For a game represented as embedded MC-nets, $E F$-value can be computed in polynomial time.

Sketch of proof. From Lemma 2 it is enough to consider hybrid rules that satisfy condition $(*)$ with weight 1 . Fix such a hybrid rule $\gamma$ and consider $G^{\gamma}$. If $V^{*} \backslash\left\{v_{1}\right\} \neq \emptyset$, i.e., there exists a node, other than $v_{1}$, with the size of a label larger than one, then from Lemma 4 it must be adjacent to $v_{1}$; hence, $E F_{i}\left(g^{\gamma}\right)=0$ for every $i \in N$. Assume otherwise. We get that $V^{*} \cup\left\{v_{1}\right\}=\left\{v_{1}\right\}$, and $U$ is the set of nodes not adjacent to $v_{1}$. From Lemma 4 we know that $U$ is an independent set. Thus, $E F_{i}\left(g^{\gamma}\right)=\sum_{S \subseteq U} \zeta_{l\left(S \cup\left\{v_{1}\right\}\right)}^{i}$ which can be computed in polynomial time.

Theorem 9. For a game represented as weighted MC-nets, computing EF-value is \#P-complete.

Sketch of proof. To show that the problem is \#P-complete, we use a Turing reduction from the problem of counting all independent sets in a graph which is \#P-complete (Valiant 1979). We use a technique described at the beginning of the section (see Eq. (5)). Specifically, for an arbitrary graph $G=$ $(V, E)$, we label each node with one player and add a new node, $v_{1}$, labeled with $j+1$ new players. We get that $U=V$ and for $i \in l\left(v_{1}\right)$ Eq. (7) iterates over independent sets in $G$ and weights depends only their size and parameter $j$.

\section{Computing HY-value}

HY-value is the first value considered by us with non-zero weights of every embedded coalition. Let us recall these weights: $\omega_{i}(S, P)=\zeta_{S}^{i} \cdot \theta(S, P) /|\mathcal{P}|$, where $\theta(S, P)=$ $\left|\left\{P^{\prime} \in \mathcal{P}:\left\{T \backslash S: T \in P^{\prime}\right\}=P \backslash\{S\}\right\}\right|$. To put it in words, $\theta(S, P)$ is the number of partitions that can be obtained from $P \backslash\{S\}$ by inserting players from $S$.

Let us introduce a notion of Bell numbers. The $n$-th Bell number, denoted $B_{n}$, is the number of all possible partitions of $n$ elements. Now, $r$-Bell numbers are a generalization of Bell numbers: $B_{n, r}$ is the number of partitions of $n+r$ elements such that the first $r$ elements are in distinct subsets (Mezo 2011). In particular, $B_{n, 0}=B_{n}=B_{n-1,1}$.

Now, observe that $\theta(S, P)=B_{|S|,|P|-1}$. Thus, HY-value weights combined with Eq. (4) yields:

$$
H Y_{i}\left(g^{\gamma}\right)=\frac{1}{B_{n}} \sum_{f \in C_{k}\left(G^{\gamma}\right)} \frac{\zeta_{l\left(S_{f}^{1}\right)}^{i}}{\theta(f)} \cdot B_{\left|l\left(S_{f}^{1}\right)\right|,\left|l\left(P_{f}\right)\right|-1} .
$$

Thus, for a fixed player $i$ and size of $l\left(S_{f}^{1}\right)$, the weight of a coloring depends solely on the number of colors it uses (see Table 2 for an illustration).

We will prove that computing this sum is \#P-complete.

Theorem 10. For a game represented as embedded $M C$ nets or weighted MC-nets, computing HY-value is \#Pcomplete.

Sketch of proof. To show that the problem is \#P-complete, we use the (Turing) reduction from the chromatic polynomial problem, i.e., counting $m$-colorings in a graph, which is \#P-complete (Jaeger, Vertigan, and Welsh 1990).

Let $G=(V, E)$ be an arbitrary graph and $c_{m}$ be the number of $k$-colorings that use exactly $m$ colors. For every $j \in\{1, \ldots, k\}$ we construct a graph $G^{\gamma_{j}}=\left(V \cup\left\{v_{1}\right\}, E \cup\right.$ $\left.\left\{\left\{v_{1}, v_{i}\right\}: v_{i} \in V\right\}\right)$ with $l\left(v_{i}\right)=\{i\}$ for $v_{i} \in V$ and $l\left(v_{1}\right)=\{1\} \cup\{k+2, \ldots, k+j\}$. Since node $v_{1}$ is connected to all other nodes we know that $G^{\gamma_{j}}$ satisfies conditions from Lemma 4 and from Lemma 2 there exists a hybrid rule $\gamma_{j}$ equivalent to some embedded MC-nets rule such that $G^{\gamma_{j}}$ is the corresponding graph. Now, it can be shown that

$$
H Y_{1}\left(g^{\gamma_{j}}\right)=\frac{(j-1) !}{(k+j) ! B_{k+j}} \sum_{m=1}^{k}(k-m) ! B_{j, m} c_{m}
$$

In result, we get a system of linear equations from Eq. (5) where $a_{j, m}=(k-m) ! B_{j, m}, x_{m}=c_{m}$ and $f(j)=((k+$ $j) ! /(j-1) !) B_{k+j} H Y_{1}\left(g^{\gamma_{j}}\right)$.

To show that the determinant of $A=\left(a_{j, m}\right)_{1 \leq j, m \leq k}$ is non-zero we first prove a general formula for $r$-Bell numbers (Lemma 11) and then, using this formula, we prove that the determinant of matrix $\left(B_{j, m}\right)_{1 \leq j, m \leq k}$ is equal to the known determinant of matrix $\left(B_{j+m}\right)_{1 \leq j, m \leq k}$ (Lemma 12).

Lemma 11. For every $n, r \in \mathbb{N}, B_{n+r}=\sum_{i=1}^{r}\{r, i\} B_{n, i}$, where $\{r, i\}$ is the Stirling number of the second kind, i.e., the number of partitions of $r$ elements into $i$ subsets.

Lemma 12. The determinant of matrix $B=\left(B_{j, m}\right)_{1 \leq j, m \leq k}$ equals $\left(\prod_{i=0}^{k} i !\right) \cdot\left(\sum_{i=0}^{k} 1 / i !\right)$.

This concludes the proof. 


\section{Computing SS-value}

SS-value, considered next, is probably the most popular extended Shapley value. Weights of SS-value combined with Eq. (4) yields:

$$
S S_{i}\left(g^{\gamma}\right)=\sum_{f \in C_{k}\left(G^{\gamma}\right)} \frac{\zeta_{l\left(S_{f}^{1}\right)}^{i}}{\theta(f)} \frac{\prod_{T \in P_{f} \backslash\left\{S_{f}^{1}\right\}}(|l(T)|-1) !}{\left(n-\left|l\left(S_{f}^{1}\right)\right|\right) !} .
$$

In what follows, let us focus on graphs in which every node is labeled with a single player: $|l(v)|=1$ for every $v \in V$. In such a case, we have $n=k=|V|$ and $|l(T)|=$ $|T|$. Under this assumption, formula for SS-value of player $i \in l\left(v_{1}\right)$ is as follows:

$$
S S_{i}\left(g^{\gamma}\right)=\sum_{f \in C_{k}\left(G^{\gamma}\right)} \frac{\prod_{T \in P_{f}}(|T|-1) !}{\theta(f) \cdot n !} .
$$

For a fixed partition $P \in \mathcal{P}$, value $\prod_{T \in P}(|T|-1)$ ! is the number of permutations in which $P$ is the partition obtained from a cycle decomposition (for a permutation $h: N \rightarrow N$ such partition is defined as follows: $\{\{i, h(i), h(h(i)), \ldots\}$ : $i \in N\}$ ). Hence, Eq. (9) is the probability that the partition obtained from a cycle decomposition of a random permutation corresponds to a (proper vertex) coloring in graph $G^{\gamma}$.

Let us consider a complement of graph $G^{\gamma}=(V, E)$ :

$$
\overline{G^{\gamma}}=(V,\{\{v, u\}: v, u \in V, v \neq u\} \backslash E) .
$$

For every coloring $f \in C_{k}\left(G^{\gamma}\right)$, sets of nodes in $P_{f}$ are independent sets in $G^{\gamma}$. Hence, they are cliques in $\overline{G^{\gamma}}$. As a consequence, we get that $S S_{i}\left(g^{\gamma}\right)$ from Eq. (9) is equivalently a weighted sum over clique covers (i.e., partitions of the nodes in a graph into cliques):

$$
S S_{i}\left(g^{\gamma}\right)=\frac{1}{n !} \sum_{P \in Q C\left(\overline{G^{\gamma}}\right)} \prod_{T \in P}(|T|-1) !,
$$

where $Q C(G)$ is the set of all clique covers in graph $G$.

Now, assume that $\overline{G^{\gamma}}$ is a bipartite graph. In such a case, there are no cliques of size larger than 2 and each partition into cliques is equivalent to a matching (not necessary perfect or maximal) in this graph. Moreover, for each such a partition we have $\prod_{T \in P}(|T|-1) !=1$ (see also Table 2 ). Hence, Eq. (10) is equal to the number of matchings in $\overline{G^{\gamma}}$ divided by $n$ !. We formalize this reasoning in the following theorem.

Theorem 13. For a game represented as embedded $M C$ nets or weighted MC-nets, computing SS-value is \#Pcomplete.

Sketch of proof. To show that the problem is \#P-complete, we use a reduction from the problem of counting all matchings in a bipartite graph which is \#P-complete (Valiant 1979). For an arbitrary bipartite graph $G=(V, E)$ we construct a graph $\overline{G^{\gamma}}$ by adding a new node, $v_{1}$, and we label each node with one player. Since node $v_{1}$ does not have any edges in $\overline{G^{\gamma}}$, then it is connected to all nodes in $G^{\gamma}$; hence, $G^{\gamma}$ satisfies conditions of Lemma 4 and from Lemma 2 there exist a hybrid rule $\gamma$ equivalent to some embedded MC-nets rule such that $G^{\gamma}$ is the corresponding graph.

Now, from Eq. (10) for $i \in l\left(v_{1}\right)$ we get that $S S_{i}\left(g^{\gamma}\right) / n$ ! equals the number of matchings in $\overline{G^{\gamma}}$, so also in $G$.

\section{Computing MY-value}

The last value that we consider is MY-value which is the first chronologically proposed extension of the Shapley value. Eq. (4) for weights of MY-value gives:

$$
M Y_{i}\left(g^{\gamma}\right)=\sum_{f \in C_{k}\left(G^{\gamma}\right)} \frac{(-1)^{\left|P_{f}\right|}\left(\left|P_{f}\right|-2\right) !}{\theta(f)} \cdot h_{i}(f),
$$

with $h_{i}(f)=\left(\sum_{T \in P_{f} \backslash\left\{S_{f}^{1}\right\}, i \notin l(T)} \frac{1}{(n-|l(T)|)}\right)-\frac{\left|P_{f}\right|-1}{n}$. From Table 2 it is visible that both techniques used for HYvalue and SS-value does not work in this case.

To cope with this problem, we will exploit the fact that weights of MY-value have a form of a sum over all coalitions. Specifically, we will consider a difference between the MY-value of two players. Let us denote such difference for players $i$ and $j$ in game $g$ by $\Delta_{i}^{j}(g): \Delta_{i}^{j}(g)=$ $M Y_{i}(g)-M Y_{j}(g)$. Now, for $i \in l\left(S_{f}^{1}\right)$ and $j \in l(T)$ for some $T \in P_{f} \backslash\left\{S_{f}^{1}\right\}$ we get $h_{i}(f)-h_{j}(f)=1 /(n-|T|)$ and

$$
\Delta_{i}^{j}\left(g^{\gamma}\right)=\sum_{f \in C_{k}\left(G^{\gamma}\right)} \frac{(-1)^{\left|P_{f}\right|}\left(\left|P_{f}\right|-2\right) !}{\theta(f) \cdot(n-|l(T)|)} .
$$

Note that if $j$ is in a label of a node adjacent to all other nodes, then the weight of a coloring depends solely on the number of colors it uses. Hence, we can use a technique described at the beginning of this section (see Eq. (5)).

Theorem 14. For a game represented as embedded $M C$ nets or weighted MC-nets, computing MY-value is \#Pcomplete.

Sketch of proof. To show that the problem is \#P-complete, again we use the reduction from the problem of counting all matching in a bipartite graph which is \#P-complete (Valiant 1979). With the same reasoning as in SS-value, instead of considering colorings in graph $G^{\gamma}$ we will focus on clique covers in the complement graph $\overline{G^{\gamma}}$. For an arbitrary bipartite graph $G=(V, E)$ for $j \in\{1, \ldots, k\}$, we construct a graph $\overline{G^{\gamma_{j}}}$ by adding $j+2$ isolated nodes and consider $\Delta_{i}^{j}$ for two players from newly added nodes. In this way, based on Eq. (11) we build a system of linear equations, as in Eq. (5).

\section{Conclusions}

In this paper, we studied the complexity of computing extended Shapley value in games represented as embedded and weighted MC-nets. Our results show that weighted MCnets, which are more concise than embedded MC-nets, are slightly worse when it comes to the Shapley value computation. Also, combined with the work by Skibski et al. (2020), we get that computational properties of partition decision trees are significantly better than both MC-nets representations.

There are many possible directions of further research. The extended Shapley value proposed by Bolger (1989), as well as other solution concepts can be considered. Also, it would be interesting to analyze hybrid rules and corresponding graphs not as a representation, but as a graph-restriction scheme for games with externalities (Myerson 1977a). 


\section{Acknowledgments}

Oskar Skibski was supported by the Polish National Science Centre grant 2015/19/D/ST6/03113.

\section{References}

Abe, T., and Funaki, Y. 2017. The non-emptiness of the core of a partition function form game. International Journal of Game Theory 46(3):715-736.

Aziz, H.; Lachish, O.; Paterson, M.; and Savani, R. 2009. Power indices in spanning connectivity games. In Algorithmic Aspects in Information and Management. Springer. 5567.

Bolger, E. M. 1989. A set of axioms for a value for partition function games. International Journal of Game Theory 18(1):37-44.

Chalkiadakis, G.; Elkind, E.; and Wooldridge, M. 2011. Computational aspects of cooperative game theory. Synthesis Lectures on Artificial Intelligence and Machine Learning 5(6):1-168.

De Clippel, G., and Serrano, R. 2008. Marginal contributions and externalities in the value. Econometrica 76(6):1413-1436.

Dunne, P. E. 2005. Multiagent resource allocation in the presence of externalities. In Multi-Agent Systems and Applications IV. Springer. 408-417.

Feldman, B. E. 1996. Bargaining, coalition formation, and value. Ph.D. Dissertation, State University of New York at Stony Brook.

Hu, C.-C., and Yang, Y.-Y. 2010. An axiomatic characterization of a value for games in partition function form. SERIEs 1(4):475-487.

Ieong, S., and Shoham, Y. 2005. Marginal contribution nets: A compact representation scheme for coalitional games. In Proceedings of the 6th ACM Conference on Electronic Commerce (ACM-EC), 193-202.

Jaeger, F.; Vertigan, D. L.; and Welsh, D. J. 1990. On the computational complexity of the Jones and Tutte polynomials. Mathematical Proceedings of the Cambridge Philosophical Society 108(1):35-53.

Kóczy, L. Á. 2018. The Shapley-value. In Partition Function Form Games: Coalitional Games with Externalities. Springer. 173-200.

Macho-Stadler, I.; Pérez-Castrillo, D.; and Wettstein, D. 2007. Sharing the surplus: An extension of the Shapley value for environments with externalities. Journal of Economic Theory 135(1):339-356.

McQuillin, B. 2009. The extended and generalized Shapley value: Simultaneous consideration of coalitional externalities and coalitional structure. Journal of Economic Theory 144(2):696-721.

Mezo, I. 2011. The r-Bell numbers. Journal of Integer Sequences 14(2):3.

Michalak, T. P.; Marciniak, D.; Szamotulski, M.; Rahwan, T.; Wooldridge, M.; McBurney, P.; and Jennings, N. R. 2010a. A logic-based representation for coalitional games with externalities. In Proceedings of the 9th International Conference on Autonomous Agents and Multiagent Systems (AAMAS), 125-132.

Michalak, T. P.; Rahwan, T.; Marciniak, D.; Szamotulski, M.; and Jennings, N. R. 2010b. Computational aspects of extending the Shapley value to coalitional games with externalities. In Proceedings of the 19th European Conference on Artificial Intelligence (ECAI), 197-202.

Michalak, T. P.; Rahwan, T.; Szczepański, P. L.; Skibski, O.; Narayanam, R.; Wooldridge, M.; and Jennings, N. R. 2013. Computational analysis of connectivity games with applications to the investigation of terrorist networks. In Proceedings of the 23rd International Joint Conference on Artificial Intelligence (IJCAI), 293-301.

Myerson, R. B. 1977a. Graphs and cooperation in games. Mathematical Methods of Operations Research 2(3):225229.

Myerson, R. B. 1977b. Values of games in partition function form. International Journal of Game Theory 6:23-31.

Pham Do, K. H., and Norde, H. 2007. The Shapley value for partition function form games. International Game Theory Review 9(02):353-360.

Rahwan, T.; Michalak, T. P.; Wooldridge, M.; and Jennings, N. R. 2012. Anytime coaliton structure generation in multiagent systems with positive or negative externalities. Artificial Intelligence 186(0):95-122.

Shapley, L. S. 1953. A value for n-person games. In Kuhn, H., and Tucker, A., eds., Contributions to the Theory of Games, volume II. Princeton University Press. 307-317.

Skibski, O.; Matejczyk, S.; Michalak, T.; Wooldridge, M.; and Yokoo, M. 2016. k-Coalitional cooperative games. In Proceedings of the 15th International Conference on Autonomous Agents and Multiagent Systems (AAMAS), 177185.

Skibski, O.; Michalak, T. P.; Sakurai, Y.; Wooldridge, M.; and Yokoo, M. 2020. Partition decision trees: A representation for efficient computation of the Shapley value extended to games with externalities. Autonomous Agents and MultiAgent Systems.

Skibski, O.; Michalak, T. P.; and Wooldridge, M. 2018. The Stochastic Shapley value for games with externalities. Games and Economic Behavior 108:65-80.

Valiant, L. G. 1979. The complexity of enumeration and reliability problems. SIAM Journal on Computing 8(3):410421.

Yi, S.-S. 2003. Endogenous formation of economic coalitions: A survey on the partition function approach. In The Endogenous Formation of Economic Coalitions. London, UK: Edward Elgar. 80-127. 